\title{
D'ALEMBERT'S AND WILSON'S FUNCTIONAL EQUATIONS FOR VECTOR AND $2 \times 2$ MATRIX VALUED FUNCTIONS
}

\author{
HENRIK STETK ÆR
}

\begin{abstract}
We solve generalizations of d'Alembert's and Wilson's functional equations where the functions to be determined are defined on an abelian group $G$ and take their values in $\mathrm{C}^{2}$ or in the complex $2 \times 2$ matrices. The solution formulas are expressed in the terms of the harmonic analysis on $G$ : Complex characters, and additive and quadratic maps of $G$ into C.
\end{abstract}

\section{Introduction}

We solve certain functional equations where the unknowns are continuous functions defined on an abelian topological group $(G,+)$ and taking their values in $\mathrm{C}^{2}$ or in the algebra $M_{2}(\mathrm{C})$ of complex $2 \times 2$ matrices. In doing so we generalize results on classical functional equations like d'Alembert's (also called the cosine functional equation)

$$
\frac{\phi(x+y)+\phi(x-y)}{2}=\phi(x) \phi(y), \quad x, y \in \mathrm{R},
$$

in which $\phi \in C(\mathrm{R})$ is the unknown, and Wilson's generalization [24] from 1919 of it

$$
\frac{f(x+y)+f(x-y)}{2}=f(x) \phi(y), \quad x, y \in \mathrm{R},
$$

in which $f, \phi \in C(\mathrm{R})$ are the unknowns.

We let $\sigma: G \rightarrow G$ be a continuous homomorphism such that $\sigma^{2}=I$. Here and throughout the paper $I$ denotes the identity operator or identity matrix, depending on the context.

Extending from the classical case of $\mathrm{R}$ to $G$ Kannappan [12] and Baker [4] found that the non-zero solutions $\phi \in C(G)$ of d'Alembert's functional equation 


$$
\frac{\phi(x+y)+\phi(x+\sigma y)}{2}=\phi(x) \phi(y), x, y \in G,
$$

are the functions of the form $\phi=(\gamma+\gamma \circ \sigma) / 2$, where $\gamma$ ranges over the continuous homomorphisms of $G$ into $\mathrm{C}^{*}=(\mathrm{C} \backslash\{0\}, \cdot)$. Classically $\sigma=-I$ so the non-zero solutions of (1) are the functions of the form $\phi(x)=(\exp (i \lambda x)+\exp (-i \lambda x)) / 2$ where $\lambda \in \mathrm{C}$. We shall in this paper carry their extension still further by writing down the solutions of the functional equation

$$
\frac{\Phi(x+y)+\Phi(x+\sigma y)}{2}=\Phi(y) \Phi(x), \quad x, y \in G,
$$

where the function $\Phi$ to be determined takes its values in $M_{2}(\mathrm{C})$. We will here still call (4) d'Alembert's functional equation.

Noting that $\Phi(0): C^{2} \rightarrow C^{2}$ is a projection the solutions of (4) can be described roughly as follows: If $\Phi(0)=I$ then there is a $C \in \mathrm{GL}(2, \mathrm{C})$ such that all the matrices $C \Phi(x) C^{-1}, x \in G$, are upper triangular. Their diagonal elements $\phi_{1}$ and $\phi_{2}$ are solutions of the scalar d'Alembert functional equation (3) so they have the form $\phi_{j}=\left(\gamma_{j}+\gamma_{j} \circ \sigma\right) / 2, j=1,2$, where $\gamma_{1}, \gamma_{2}: G \rightarrow C^{*}$ are continuous homomorphisms. The upper right hand corner is 0 if $\phi_{1} \neq \phi_{2}$, and explicit formulas are given in Theorem 2.3 in the degenerate case of $\phi_{1}=\phi_{2}$. If $\Phi(0) \neq I$, then $\Phi(0)$ is a projection of dimension 1 or 0 , so we are once more led to a scalar functional equation. Proposition 2.5 states what the solutions are. The solution formulas of (4) and the functional equation (5) below are expressed in terms of $\sigma$ and basic building blocks of harmonic analysis on $G$ : Complex characters, and additive and quadratic maps of $G$ into $\mathrm{C}$.

The results obtained for d'Alembert's functional equation (4) enable us to solve a mixed vector-matrix analogue of Wilson's functional equation (2), viz.

$$
\frac{g(x+y)+g(x+\sigma y)}{2}=\Phi(y) g(x), \quad x, y \in G,
$$

where the unknown function $g: G \rightarrow \mathrm{C}^{2}$ is a continuous vector valued function (column vector) and the other unknown function $\Phi: G \rightarrow M_{2}(\mathrm{C})$ is a continuous matrix valued function. It turns out that (5) has only 6 different types of solutions plus 2 degenerate types (Corollary 3.6). The crucial relation between (4) and (5) is observed in Proposition 3.1(2): $g, \Phi$ is a solution of (5) and the two component functions of $g$ are linearly independent then $\Phi$ satisfies (4). The observation has roots back to Wilson $[24 ; \S 6]$ and Kaczmarz [11].

Throughout the paper we deal with topological abelian groups and con- 
tinuous mappings, but we can of course apply the results to any abelian group $G$ by giving it the discrete topology. However, there is a point in taking the continuity into consideration. To take an example, let $\phi$ be a nonzero continuous solution of d'Alembert's functional equation (3). Then as mentioned above $\phi$ has the form $\phi=(\gamma+\gamma \circ \sigma) / 2$, where $\gamma: G \rightarrow \mathrm{C}^{*}$ is a continuous homomorphism. If we discard the topology on $G$ and only work with the discrete topology then we do not know that $\gamma$ is continuous when $\phi$ is. To infer that would require separate arguments.

We can obtain the solutions to the scalar equations from the matrix and vector valued equations by working with diagonal matrix functions and vector functions whose components are identical. On the other hand we use the scalar results in deriving ours.

Let us describe how our work relates to the literature:

In most of it only the cases $\sigma=-I$ (d'Alembert type functional equations) and $\sigma=I$ (Levi-Civita functional equations) have been discussed. Furthermore the two cases were treated separately. We give a unified treatment, valid for general involutive automorphisms $\sigma$, so our discussion comprises not only $\sigma= \pm I$, but also examples like the reflection in a hyperplane of $\mathrm{R}^{n}$ and the symplectic involution of the additive group of $2 \times 2$ matrices. New phenomena occur outside the situation of $G=2 G$ and $\sigma=-I$, causing our solution formulas to be more involved than the earlier ones: We have to take homomorphisms $\gamma: G \rightarrow \mathbf{C}^{*}=(\mathbf{C} \backslash\{0\}, \cdot)$ for which $\gamma=\gamma \circ \sigma$ into account. When $G=2 G$ and $\sigma=-I$ the only such homomorphism is $\gamma=1$.

The treatment by Sinopoulos [18] of the two functional equations (4) and (5) was the point of departure of the present paper. Our purpose is threefold: (a) To get rid of the assumption $G=2 G$ that occurs in most of the literature and also in [18], (b) as far as possible to avoid Sinopoulos' special assumption that the components of the vector valued function $g$ in (5) are linearly independent, and (c) to derive the results for general involutive automorphisms $\sigma: G \rightarrow G$, not just for $\sigma=-I$.

The matrix or even operator version (4) of d'Alembert's functional equation with $\sigma=-I$ has for $\Phi(0)=I$ been treated by Fattorini [9], Kurepa [15], [16], Baker and Davison [5], Kisyński [13], [14], Székelyhidi [22], Chojnacki [6], [8], Sinopoulos [18] and (for general $\sigma$ ) the author [20], under various conditions like $G=\mathrm{R}$ or just $G=2 G$, or the solution $\Phi$ being bounded on $G$. As mentioned we here do without these conditions for solutions of (4) taking their values in the algebra $M_{2}(\mathrm{C})$. We also find the solutions of (4) when $\Phi(0) \neq I$ (Proposition 2.5); these formulas have not been written down in the literature.

Wilson's functional equation seems not to have been studied in the mixed vector-matrix form (5) prior to Sinopoulos' paper [18], in which $\sigma=-I$. In 
contrast to [18] we work with a general involutive automorphism $\sigma$ and do not need extra assumptions on the group $G$ and the solutions of (5).

The scalar d'Alembert functional equation (3) was for a general abelian group $G$ first solved by Kannappan [12] who worked with $\sigma=-I$. Its solution for an arbitrary involutive automorphism $\sigma$ was written down by Baker [4] (see also Theorem 3.1 of [21]). Badora [3], Gajda [10] and Chojnacki [7] have studied bounded solutions of certain scalar generalizations of (3).

Many other scalar functional equations have been discussed. The monographs by Aczél [1] and by Aczél and Dhombres [2] contain many references.

In addition to the terminology introduced above we shall need the following notation.

Notation. We let 0 denote the neutral element of the abelian topological group $(G,+)$. The algebra of all continuous complex valued functions on $G$ is denoted $C(G)$. We let $\mathscr{M}(G)$ be the set of all continuous homomorphisms $\gamma: G \rightarrow \mathrm{C}^{*}=(\mathrm{C} \backslash\{0\}, \cdot)$, and put $\mathscr{M}^{+}(G):=\{\gamma \in \mathscr{M}(G) \mid \gamma \circ \sigma=\gamma\}$. We let $\mathscr{A}(G)$ be the set of all continuous additive maps of $G$ into $(\mathrm{C},+)$, and let $\mathscr{A}^{ \pm}(G)=\{a \in \mathscr{A}(G) \mid a \circ \sigma= \pm a\}$. We let $\mathscr{S}(G)$ be the set of maps $S: G \rightarrow$ C of the form $S(x)=q(x, x), x \in G$, where $q: G \times G \rightarrow$ C ranges over the continuous bi-additive symmetric maps, and let $\mathscr{S}^{-}(G)$ be the subset for which $q$ satisfies $q(\sigma x, y)=-q(x, y)$ for all $x, y \in G$.

The transpose of a matrix $A$ is denoted by $A^{t}$.

Finally we view the elements of $\mathrm{C}^{n}$ as $n \times 1$ matrices, i.e. as column vectors.

In the classical instance of $\sigma=-I$ there are some simplifications: $\mathscr{A}^{+}(G)=\{0\}, \mathscr{A}^{-}(G)=\mathscr{A}(G)$ and $\mathscr{S}^{-}(G)=\mathscr{S}(G)$. If furthermore $2 G=G$ then $\mathscr{M}^{+}(G)=\{1\}$.

\section{2. d'Alembert's functional equation for $2 \times 2$ matrices}

In this section we write down the set of solutions of the matrix functional equation

$$
\frac{\Phi(x+y)+\Phi(x+\sigma y)}{2}=\Phi(y) \Phi(x), \quad x, y \in G,
$$

for continuous $\Phi: G \rightarrow M_{2}(\mathrm{C})$. The knowledge of the solutions of (6) is crucial for our study in Section 3 below of the functional equation (5).

We can change the order $\Phi(y) \Phi(x)$ of the factors on the right hand side of (6) by working with the transpose $\Phi^{t}$ instead of $\Phi$. Then it becomes the cosine matrix equation studied by Székelyhidi [22].

To set the stage let us mention that Chojnacki [6] proved that the $\Phi(x), x \in G$, for a bounded solution $\Phi$ of the cosine equation 
D'ALEMBERT'S AND WILSON'S FUNCTIONAL EQUATIONS FOR VECTOR... 119

$$
\frac{\Phi(x+y)+\Phi(x-y)}{2}=\Phi(x) \Phi(y), \quad x, y \in G,
$$

such that $\Phi(0)=I$ can be simultaneously diagonalized, a fact that is false in the unbounded case even for $G=\mathrm{R}$ (see [13]). We do not wish to restrict ourselves to bounded solutions so the best we can hope for is that the matrices $\Phi(x), x \in G$, of (6) can be brought into triangular form simultaneously and their forms then be explicitly found. We will in this Section 2 show how this program to a large extent can be realized.

Putting $y=0$ in (6) we get $\Phi=\Phi(0) \Phi$, from which we see that $\Phi(0): \mathrm{C}^{2} \rightarrow \mathrm{C}^{2}$ is a projection. So there are only the following three possibilities: $\Phi(0)=I, \Phi(0)$ is a 1-dimensional projection, or $\Phi(0)=0$. However, the last possibility is uninteresting because it implies that $\Phi=\Phi(0) \Phi=0$. The case of $\Phi(0)=I$ is the contents of Theorem 2.3, while the case of $\Phi(0)$ being a 1-dimensional projection is solved in Proposition 2.5 below.

We do not need the assumptions $\sigma=-I, G=2 G$ and $\Phi(0)=I$ that the articles cited above impose.

2.1. Proposition. If $\Phi: G \rightarrow M_{2}(\mathrm{C})$ satisfies (6) and $\Phi(0)=I$, then $\Phi=\Phi \circ \sigma$ and $\Phi(x) \Phi(y)=\Phi(y) \Phi(x)$ for all $x, y \in G$.

Proof. Putting $x=0$ in (6) we see that $\Phi=\Phi \circ \sigma$. Now

$$
\begin{aligned}
\Phi(x) \Phi(y) & =\frac{1}{2}\{\Phi(y+x)+\Phi(y+\sigma x)\}=\frac{1}{2}\{\Phi(x+y)+\Phi \circ \sigma(x+\sigma y)\} \\
& =\frac{1}{2}\{\Phi(x+y)+\Phi(x+\sigma y)\}=\Phi(y) \Phi(x) .
\end{aligned}
$$

Counterexamples reveal that the assumption $\Phi(0)=I$ cannot be deleted in Proposition 2.1 (see Remarks 2.6 below).

For later reference we state as Theorem 2.2 what the solutions of the scalar d'Alembert's and Wilson's functional equations are. The proof of it can be found in [21; Theorem 3.1 and Theorem 3.4].

\subsection{THEOREM.}

1. Let $\phi \in C(G)$ be a nonzero solution of d'Alembert's functional equation

$$
\frac{\phi(x+y)+\phi(x+\sigma y)}{2}=\phi(x) \phi(y), \quad x, y \in G .
$$

Then there exists $\gamma \in \mathscr{M}(G)$ such that $\phi=(\gamma+\gamma \circ \sigma) / 2$.

2. If Wilson's functional equation

$$
\frac{f(x+y)+f(x+\sigma y)}{2}=f(x) \phi(y), \quad x, y \in G,
$$

has a solution $f, \phi \in C(G)$ with $f \neq 0$, then $\phi$ is a nonzero solution of d'Alembert's functional equation (9). 
3. Let $f, \phi \in C(G)$ be a solution of Wilson's functional equation (10) with $\phi=(\gamma+\gamma \circ \sigma) / 2$ for some $\gamma \in \mathscr{M}(G)$. Then $f$ may be written in the form

$$
\begin{aligned}
& f=c(\gamma+\gamma \circ \sigma) / 2+c^{\prime}(\gamma-\gamma \circ \sigma) / 2 \text { if } \gamma \neq \gamma \circ \sigma, \text { and } \\
& f=\gamma\left(c+a^{-}\right) \text {if } \gamma=\gamma \circ \sigma,
\end{aligned}
$$

where $c, c^{\prime} \in \mathrm{C}$ and $a^{-} \in \mathscr{A}^{-}(G)$.

The following Theorem 2.3 is one of the main results of the present paper. It gives information about the form of the solutions $\Phi$ of the functional equation (6). Theorem 3.3 below reveals a further fine structure of the solutions.

2.3. TheOREM. The continuous solutions $\Phi: G \rightarrow M_{2}(\mathrm{C})$ of the matrix functional equation (6) satisfying $\Phi(0)=I$ are the matrix valued functions of the three forms below in which $C$ ranges over $\mathrm{GL}(2, \mathrm{C})$ :

1.

$$
\Phi=C\left\{\begin{array}{cc}
\left(\gamma_{1}+\gamma_{1} \circ \sigma\right) / 2 & 0 \\
0 & \left(\gamma_{2}+\gamma_{2} \circ \sigma\right) / 2
\end{array}\right\} C^{-1}
$$

where $\gamma_{1}, \gamma_{2} \in \mathscr{M}(G)$.

2.

$$
\Phi=C\left\{\begin{array}{cc}
\gamma+\gamma \circ \sigma) / 2 & (\gamma+\gamma \circ \sigma) a^{+} / 2+(\gamma-\gamma \circ \sigma) a^{-} / 2 \\
0 & (\gamma+\gamma \circ \sigma) / 2
\end{array}\right\} C^{-1}
$$

where $\gamma \in \mathscr{M}(G)$ has $\gamma \neq \gamma \circ \sigma$ and where $a^{ \pm} \in \mathscr{A}^{ \pm}(G)$.

3.

$$
\Phi=C \gamma^{+}\left\{\begin{array}{cc}
1 & a^{+}+S^{-} \\
0 & 1
\end{array}\right\} C^{-1}
$$

where $\gamma^{+} \in \mathscr{M}^{+}(G), a^{+} \in \mathscr{A}^{+}(G)$ and $S^{-} \in \mathscr{S}^{-}(G)$. We may here under (3) assume that $a^{+}+S^{-} \neq 0$.

Proof of Theorem 2.3. It is easy to check that the all of the possibilities listed in Theorem 2.3 define solutions of (6), so it is left to show that each solution $\Phi$ has one of the listed forms.

The matrices $\Phi(x), x \in G$, commute with one another (Proposition 2.1), so by linear algebra there is a matrix $C \in \operatorname{GL}(2, \mathrm{C})$ such that

$$
C^{-1} \Phi(x) C=\left\{\begin{array}{cc}
\phi_{1}(x) & \psi(x) \\
0 & \phi_{2}(x)
\end{array}\right\}, x \in G .
$$

Furthermore, if $\phi_{1} \neq \phi_{2}$ then we may assume that $\psi=0$.

The matrix identity (6) implies that $\phi_{1}$ and $\phi_{2}$ both satisfy the scalar 
d'Alembert's functional equation (3). According to Theorem 2.2 there exist $\gamma_{i} \in \mathscr{M}(G)$ such that $\phi_{i}=\left(\gamma_{i}+\gamma_{i} \circ \sigma\right) / 2$ for $i=1,2$. If $\phi_{1} \neq \phi_{2}$ then we may assume that $\psi=0$, so we deal with case (1) of Theorem 2.3. From now on we may assume that $\phi_{1}=\phi_{2} \equiv \phi$ and that $\gamma_{1}=\gamma_{2} \equiv \gamma$. The identity (6) implies that

$$
\frac{\psi(x+y)+\psi(x+\sigma y)}{2}=\phi(x) \psi(y)+\psi(x) \phi(y), \quad x, y \in G .
$$

Proposition 3.7 of [21] gives the solutions of (16) for $\phi=(\gamma+\gamma \circ \sigma) / 2$ : If $\gamma \neq \gamma \circ \sigma$ then $\psi$ has the form

$$
\psi=\frac{\gamma+\gamma \circ \sigma}{2} a^{+}+\frac{\gamma-\gamma \circ \sigma}{2} a^{-},
$$

where $a^{ \pm} \in \mathscr{A}^{ \pm}(G)$. And if $\gamma=\gamma \circ \sigma$ then $\psi$ has the form $\psi=\gamma\left[a^{+}+S^{-}\right]$, where $a^{+} \in \mathscr{A}^{+}(G)$ and $S^{-} \in \mathscr{S}^{-}(G)$. This fits into the last 2 cases of Theorem 2.3.

2.4. REMARK. The solutions of the cosine equation (7) are well known for $G=\mathrm{R}$, even when the solution $\Phi$ takes values in a Banach algebra $\mathscr{A}$ (see Kurepa [15] and the further discussion in Baker and Davidson [5]). However, the solution formulas in the literature are expressed very differently from the way we use here: Kurepa's result, stated as Theorem 13.19 of [2], says that there exists an $A \in \mathscr{A}$ such that

$$
\Phi(x)=\sum_{n=0}^{\infty} \frac{x^{2 n}}{(2 n) !} A^{n}, \quad x \in \mathrm{R},
$$

if $\Phi: \mathrm{R} \rightarrow \mathscr{A}$ is continuous. In all three cases of Theorem 2.3 the matrix $A \in M_{2}(\mathrm{C})$ can be found explicitly:

Case I: Writing $\gamma_{i}(x)=\exp \left(\lambda_{i} x\right)$ for $i=1,2$ we get

$$
A=C\left\{\begin{array}{cc}
\lambda_{1}^{2} & 0 \\
0 & \lambda_{2}^{2}
\end{array}\right\} C^{-1} .
$$

Case II: Writing $\gamma(x)=\exp (\lambda x)$ and $a(x)=c x$ we get

$$
A=C\left\{\begin{array}{cc}
\lambda^{2} & 2 c \lambda \\
0 & \lambda^{2}
\end{array}\right\} C^{-1} .
$$

Case III: Writing $S(x)=c x^{2}$ we get

$$
A=C\left\{\begin{array}{cc}
0 & 2 c \\
0 & 0
\end{array}\right\} C^{-1} .
$$


If $\sigma=I$ so that $\Phi$ is a group homomorphism then the possibilities for $\Phi$ has been analyzed even on semigroups by McKiernan [17].

We proceed by discussing the second possibility for $\Phi(0)$, viz. that it is a 1dimensional projection.

2.5. Proposition. Let $\Phi: G \rightarrow M_{2}(\mathrm{C})$ be a continuous solution of d'Alembert's functional equation (6) such that $\Phi(0)$ is a 1-dimensional projection. Then there exist $\gamma \in \mathscr{M}(G)$ and $C \in \mathrm{GL}(2, \mathrm{C})$ such that $\Phi$ has the form

$$
\begin{aligned}
& \Phi=C\left\{\begin{array}{cc}
(\gamma+\gamma \circ \sigma) / 2 & (\gamma-\gamma \circ \sigma) / 2 \\
0 & 0
\end{array}\right\} C^{-1} \text { if } \gamma \neq \gamma \circ \sigma, \text { and } \\
& \Phi=C \gamma\left\{\begin{array}{cc}
1 & a^{-} \\
0 & 0
\end{array}\right\} C^{-1} \text { if } \gamma=\gamma \circ \sigma,
\end{aligned}
$$

where $c \in \mathrm{C}$ and $a^{-} \in \mathscr{A}^{-}(G)$. Conversely, any $\Phi$ of the form (22) is a solution of (6) such that $\Phi(0)$ is a 1-dimensional projection.

Proof of Proposition 2.5. Up to a similarity the projection $\Phi(0)$ has the form

$$
\Phi(0)=\left\{\begin{array}{ll}
1 & 0 \\
0 & 0
\end{array}\right\} .
$$

Discarding for simplicity of writing the similarity matrix we assume that $\Phi(0)$ is this matrix. Writing

$$
\Phi=\left\{\begin{array}{cc}
\phi & \psi \\
\psi_{1} & \phi_{1}
\end{array}\right\}
$$

we get putting $y=0$ in (6) that $\psi_{1}=\phi_{1}=0$. The identity (6) means that $\phi$ and $\psi$ are solutions of the scalar d'Alembert and Wilson functional equations respectively. Using the form (23) for $\Phi(0)$ we see that $\phi(0)=1$ and $\psi(0)=0$. Now the formulas of Theorem 2.2 imply Proposition 2.5.

2.6. Remarks. In contrast to the results for $\Phi(0)=I$ in Proposition 2.1 the matrices $\Phi(x), x \in G$, in Proposition 2.5 do not commute with one another, and $\Phi \neq \Phi \circ \sigma$, unless $c=0$ for $\gamma \neq \gamma \circ \sigma$, resp. $a^{-}=0$ for $\gamma=\gamma \circ \sigma$.

\section{On a mixed vector-matrix Wilson functional equation}

In this section we solve the mixed vector-matrix version (5) of Wilson's functional equation from the Introduction. We recall that we view the elements of $\mathrm{C}^{n}$ as column vectors.

The scalar version of the following Proposition 3.1(2) was derived by 
Wilson [24; §6] in 1919 and Kaczmarz [11] in 1924. It is also known in the theory of spherical functions (see, e.g., [19; Corollary 3.2]).

3.1. Proposition. Let $\Phi: G \rightarrow M_{n}(\mathrm{C})$. Let $g=\left\{g_{1}, \cdots, g_{n}\right\}^{t}: G \rightarrow \mathrm{C}^{n}$ satisfy the functional equation

$$
\frac{g(x+y)+g(x+\sigma y)}{2}=\Phi(y) g(x), \quad x, y \in G .
$$

1. $\Phi(y)\left(\operatorname{span}\left\{g(x) \in \mathrm{C}^{\mathrm{n}} \mid x \in G\right\}\right) \subseteq \operatorname{span}\left\{g(x) \in \mathrm{C}^{n} \mid x \in G\right\}$ for all $y \in G$, and the restriction $\Psi$ of $\Phi$ to $\operatorname{span}\left\{g(x) \in \mathrm{C}^{n} \mid x \in G\right\}$ is a solution of

$$
\frac{\Psi(x+y)+\Psi(x+\sigma y)}{2}=\Psi(y) \Psi(x), x, y \in G,
$$

such that $\Psi(0)=I$. Given $g$ then the identity (25) determines $\Phi$ completely on $\operatorname{span}\left\{g(x) \in \mathrm{C}^{n} \mid x \in G\right\}$.

2. If the component functions $g_{1}, \cdots, g_{n}$ of $g$ are linearly independent (or equivalently if $\operatorname{span}\left\{g(x) \in \mathrm{C}^{n} \mid x \in G\right\}$ has dimension $\left.n\right)$, then $\Phi$ is a solution of d'Alembert's functional equation (6) and $\Phi(0)=I$. Given $g$ then $\Phi$ is completely determined by the identity (25).

Proof. It follows directly from the identity (25) that $\Phi(y)$ leaves $\operatorname{span}\left\{g(x) \in \mathrm{C}^{2} \mid x \in G\right\}$ invariant. Adding the two identities that we obtain from (25) by replacing $y$ by $y+z$ and $y+\sigma z$ respectively, we find that

$$
\begin{aligned}
& \frac{g(x+y+z)+g(x+y+\sigma z)}{2}+\frac{g(x+\sigma y+z)+g(x+\sigma y+\sigma z)}{2} \\
& =[\Phi(y+z)+\Phi(y+\sigma z)] g(x) .
\end{aligned}
$$

Applying (25) twice to the left hand side of this we obtain the identity

$$
2 \Phi(z) \Phi(y) g(x)=[\Phi(y+z)+\Phi(y+\sigma z)] g(x) .
$$

This shows that $\Psi$ is a solution of the functional equation (26). Putting $x=0$ in the original functional equation (25) we see that $\Phi(0)$ equals the identity on $\operatorname{span}\left\{g(x) \in \mathrm{C}^{2} \mid x \in G\right\}$. This proves (1), and (2) is an immediate corollary of (1).

We will in the proof of Theorem 3.3 need to know the solutions of the functional equation of symmetric differences in product form (29). They are listed in Theorem 4.1 of [21] that we cite here:

3.2. THEOREM. The complete list of complex valued functions $f, g, h \in C(G)$ satisfying 


$$
\frac{f(x+y)+f(x+\sigma y)}{2}=f(x)+g(x) h(y), \quad x, y \in G,
$$

consists of the following 5 cases, where we let $a^{-}, a_{1}^{-}: G \rightarrow \mathrm{C}$ denote elements of $\mathscr{A}^{-}(G), a^{+}$of $\mathscr{A}^{+}(G)$, a of $\mathscr{A}(G), S^{-}$of $\mathscr{S}^{-}(G), \gamma: G \rightarrow \mathrm{C}^{*}$ continuous homomorphisms, and $c, c_{1}, c_{2}, c_{3} \in \mathrm{C}$ complex constants:

1. $f=a^{-}+c$, with $g=0$ and h arbitrary, or with $h=0$ and $g$ arbitrary.

2. There exists a $c \in \mathrm{C} \backslash\{0\}$ such that $g=c, h=a^{+}+S^{-}$and $f=c\left(a^{+}+S^{-}\right)+a^{-}+c_{1}$.

3. There exists an $a^{-} \in \mathscr{A}^{-}(G) \backslash\{0\}$ such that $g=a^{-}+c$, $h=a^{+}+c_{1}\left(a^{-}\right)^{2}$ and $f=c a^{+}+c c_{1}\left(a^{-}\right)^{2}+a^{+} a^{-}+\frac{1}{3} c_{1}\left(a^{-}\right)^{3}+a_{1}^{-}+c_{2}$.

4. There exists a for which $\gamma \neq \gamma \circ \sigma$ such that $g=c_{1} \gamma+c_{2} \gamma \circ \sigma$, $h=c\{(\gamma+\gamma \circ \sigma) / 2-1\}$ and $f=c\left(c_{1} \gamma+c_{2} \gamma \circ \sigma\right)+a^{-}+c_{3}$.

5. There exists a $\gamma \neq 1$ for which $\gamma=\gamma \circ \sigma$ such that $g=\gamma\left[a^{-}+c_{1}\right]$, $h=c[\gamma-1]$ and $f=c \gamma\left[a^{-}+c_{1}\right]+a_{1}^{-}+c_{2}$.

The main result of the present paper is the next one.

3.3. TheOREM. Let $\Phi: G \rightarrow M_{2}(\mathrm{C})$ be a continuous solution of d'Alembert's functional equation (6) such that $\Phi(0)=I$.

To each continuous solution $g: G \rightarrow \mathrm{C}^{2}$ of Wilson's functional equation

$$
\frac{g(x+y)+g(x+\sigma y)}{2}=\Phi(y) g(x), \quad x, y \in G,
$$

there exist $\alpha, \beta \in \mathrm{C}^{2}$ and $C \in \mathrm{GL}(2, \mathrm{C})$ such that

$$
g=C(E \alpha+E \circ \sigma \beta) \text { with } \Phi=C \frac{E+E \circ \sigma}{2} C^{-1}
$$

where $E: G \rightarrow M_{2}(\mathrm{C})$ has one of the following 6 forms in which $\gamma, \gamma_{1}, \gamma_{2} \in \mathscr{M}(G), \quad \gamma^{+}, \gamma_{1}^{+}, \gamma_{2}^{+} \in \mathscr{M}^{+}(G), \quad a \in \mathscr{A}(G), \quad a^{-}, a_{1}^{-}, a_{2}^{-} \in \mathscr{A}^{-}(G)$, $a^{+} \in \mathscr{A}^{+}(G), S^{-} \in \mathscr{S}^{-}(G)$ and $c, c_{1} \in \mathrm{C}$ :

$$
\begin{aligned}
& E_{1}=\left\{\begin{array}{cc}
\gamma_{1} & 0 \\
0 & \gamma_{2}
\end{array}\right\}, E_{2}=\left\{\begin{array}{cc}
\gamma_{1} & 0 \\
0 & \gamma_{2}^{+}\left(1+a^{-}\right)
\end{array}\right\}, \\
& E_{3}=\left\{\begin{array}{cc}
\gamma_{1}^{+}\left(1+a_{1}^{-}\right) & 0 \\
0 & \gamma_{2}^{+}\left(1+a_{2}^{-}\right)
\end{array}\right\} \\
& E_{4}=\gamma\left\{\begin{array}{ll}
1 & a \\
0 & 1
\end{array}\right\}, E_{5}=\gamma^{+}\left\{\begin{array}{cc}
1 & a+S^{-} \\
0 & 1
\end{array}\right\}, \\
& E_{6}=\gamma^{+}\left\{\begin{array}{cc}
1+a^{-} & c\left(a^{-}\right)^{3}+3 c\left(a^{-}\right)^{2}+a^{+}+a^{+} a^{-}+a_{1}^{-} \\
0 & 1+a^{-}
\end{array}\right\}
\end{aligned}
$$

$E_{1}, E_{2}, E_{3}$ from the list (32) give via the formula $\Phi=C[(E+E \circ \sigma) / 2] C^{-1}$ 
D'ALEMBERT'S AND WILSON'S FUNCTIONAL EQUATIONS FOR VECTOR... 125

the $\Phi$ from case (1) of Theorem 2.3. $E_{4}$ gives case (2), while $E_{5}$ and $E_{6}$ give case (3).

Conversely, any pair $\{g, \Phi\}$ described by (31) and (32) is a solution of Wilson's functional equation (30) such that $\Phi(0)=I$.

3.4. Remark. When $\sigma=-I, G=2 G$ the matrices from the list (32) simplify to:

$$
\begin{aligned}
& E_{1}=\left\{\begin{array}{cc}
\gamma_{1} & 0 \\
0 & \gamma_{2}
\end{array}\right\}, E_{2}=\left\{\begin{array}{cc}
\gamma_{1} & 0 \\
0 & 1+a
\end{array}\right\}, E_{3}=\left\{\begin{array}{cc}
1+a_{1} & 0 \\
0 & 1+a_{2}
\end{array}\right\} \\
& E_{4}=\gamma\left\{\begin{array}{ll}
1 & a \\
0 & 1
\end{array}\right\}, E_{5}=\left\{\begin{array}{cc}
1 & a+S \\
0 & 1
\end{array}\right\}, E_{6}=\left\{\begin{array}{cc}
1+a & c a^{3}+3 c a^{2}+a_{1} \\
0 & 1+a
\end{array}\right\} .
\end{aligned}
$$

Proof of Theorem 3.3. Substituting the expressions for $\Phi$ and $g$ from (31) into (30) we see that the statement about the converse boils down to the question of whether

$$
\frac{E_{j}(x+y)+E_{j}(x+\sigma y)}{2}=\frac{E_{j}(y)+E_{j}(\sigma y)}{2} E_{j}(x), \quad x, y \in G,
$$

for $j=1,2,3,4$. And this is a simple matter to check for the matrices $E_{j}$ given in (32), so we leave out the verification.

It remains to show that any solution $g$ of (30) has the form claimed in the Theorem. Introducing a $C \in \mathrm{GL}(2, \mathrm{C})$ from Theorem 2.3 making $\Psi:=C^{-1} \Phi C$ upper triangular we get from the functional equation (30) that $G:=C^{-1} g$ is a solution of the functional equation

$$
\frac{G(x+y)+G(x+\sigma y)}{2}=\Psi(y) G(x), \quad x, y \in G .
$$

Since $\Psi$ has the form

$$
\Psi=\left\{\begin{array}{cc}
\left(\gamma_{1}+\gamma_{1} \circ \sigma\right) / 2 & * \\
0 & \left(\gamma_{2}+\gamma_{2} \circ \sigma\right) / 2
\end{array}\right\}
$$

where $\gamma_{i} \in \mathscr{M}(G)$ for $i=1,2$ we get with the notation $G=\left\{G_{1}, G_{2}\right\}^{t}$ that $G_{2}$ satisfies the scalar Wilson's functional equation and so according to Theorem 2.2(3) has the form

$$
\begin{aligned}
& G_{2}=c_{2}\left(\gamma_{2}+\gamma_{2} \circ \sigma\right) / 2+c_{2}^{\prime}\left(\gamma_{2}-\gamma_{2} \circ \sigma\right) / 2 \text { if } \gamma_{2} \neq \gamma_{2} \circ \sigma, \text { and } \\
& G_{2}=\gamma_{2}\left(c_{2}+a_{2}^{-}\right) \text {if } \gamma_{2}=\gamma_{2} \circ \sigma
\end{aligned}
$$

for some $c_{2}, c_{2}^{\prime} \in \mathrm{C}$ and $a_{2}^{-} \in \mathscr{A}^{-}(G)$.

To get the missing information we work our way through the three possibilities for $\Psi$ listed in Theorem 2.3. We use the notation from Theorem 2.3. 
Case (1): Here

$$
\Psi=\left\{\begin{array}{cc}
\left(\gamma_{1}+\gamma_{1} \circ \sigma\right) / 2 & 0 \\
0 & \left(\gamma_{2}+\gamma_{2} \circ \sigma\right) / 2
\end{array}\right\}
$$

In this diagonal case also $G_{1}$ satisfies a scalar Wilson's functional equation and so it has according to Theorem 2.2(3) the form

$$
\begin{aligned}
& G_{1}=c_{1}\left(\gamma_{1}+\gamma_{1} \circ \sigma\right) / 2+c_{1}^{\prime}\left(\gamma_{1}-\gamma_{1} \circ \sigma\right) / 2 \text { if } \gamma_{1} \neq \gamma_{1} \circ \sigma, \text { and } \\
& G_{1}=\gamma_{1}\left(c_{1}+a_{1}^{-}\right) \text {if } \gamma_{1}=\gamma_{1} \circ \sigma
\end{aligned}
$$

for some $c_{1}, c_{1}^{\prime} \in \mathrm{C}$ and $a_{1}^{-} \in \mathscr{A}^{-}(G)$. This leaves us with 4 combinations:

If $\gamma_{1} \neq \gamma_{1} \circ \sigma$ and $\gamma_{2} \neq \gamma_{2} \circ \sigma$ then we may use

$$
E_{1}=\left\{\begin{array}{cc}
\gamma_{1} & 0 \\
0 & \gamma_{2}
\end{array}\right\}, \alpha=\frac{1}{2}\left\{\begin{array}{l}
c_{1}+c_{1}^{\prime} \\
c_{2}+c_{2}^{\prime}
\end{array}\right\}, \beta=\frac{1}{2}\left\{\begin{array}{l}
c_{1}-c_{1}^{\prime} \\
c_{2}-c_{2}^{\prime}
\end{array}\right\} .
$$

If $\gamma_{1} \neq \gamma_{1} \circ \sigma$, but $\gamma_{2}=\gamma_{2} \circ \sigma$ then we may use

$$
E_{2}=\left\{\begin{array}{cc}
\gamma_{1} & 0 \\
0 & \gamma_{2}\left(1+a_{2}^{-}\right)
\end{array}\right\}, \alpha=\frac{1}{2}\left\{\begin{array}{c}
c_{1}+c_{1}^{\prime} \\
c_{2}+1
\end{array}\right\}, \beta=\frac{1}{2}\left\{\begin{array}{c}
c_{1}-c_{1}^{\prime} \\
c_{2}-1
\end{array}\right\} .
$$

If $\gamma_{1}=\gamma_{1} \circ \sigma$, but $\gamma_{2} \neq \gamma_{2} \circ \sigma$ then we get with

$$
E=\left\{\begin{array}{cc}
\gamma_{1}\left(1+a_{1}^{-}\right) & 0 \\
0 & \gamma_{2}
\end{array}\right\}, \alpha=\frac{1}{2}\left\{\begin{array}{c}
c_{1}+1 \\
c_{2}+c_{2}^{\prime}
\end{array}\right\}, \beta=\frac{1}{2}\left\{\begin{array}{c}
c_{1}-1 \\
c_{2}-c_{2}^{\prime}
\end{array}\right\}
$$

that $C^{-1} g(x)=E(x) \alpha+E(\sigma(x)) \beta$ and $C^{-1} \Phi(x) C=\frac{1}{2}(E(x)+E(\sigma(x)))$. Unfortunately, $E$ does not occur on the list (32). However, replacing $G$ and $\Psi$ by $S G$ and $S \Psi S$ where $S=\left(\begin{array}{ll}0 & 1 \\ 1 & 0\end{array}\right)$ reduces the case to the previous one by essentially interchanging $\gamma_{1}$ and $\gamma_{2}$.

Finally, if $\gamma_{1}=\gamma_{1} \circ \sigma$ and $\gamma_{2}=\gamma_{2} \circ \sigma$ then we may use

$$
E_{3}=\left\{\begin{array}{cc}
\gamma_{1}\left(1+a_{1}^{-}\right) & 0 \\
0 & \gamma_{2}\left(1+a_{2}^{-}\right)
\end{array}\right\}, \alpha=\frac{1}{2}\left\{\begin{array}{l}
c_{1}+1 \\
c_{2}+1
\end{array}\right\}, \beta=\frac{1}{2}\left\{\begin{array}{l}
c_{1}-1 \\
c_{2}-1
\end{array}\right\} .
$$

Case (2): Here

$$
\Psi=\left\{\begin{array}{cc}
(\gamma+\gamma \circ \sigma) / 2 & (\gamma+\gamma \circ \sigma) a^{+} / 2+(\gamma-\gamma \circ \sigma) a^{-} / 2 \\
0 & (\gamma+\gamma \circ \sigma) / 2
\end{array}\right\}
$$

where $\gamma \in \mathscr{M}(G), \gamma \neq \gamma \circ \sigma$ and $a^{ \pm} \in \mathscr{A}^{ \pm}(G)$. From (37) we read that

$$
G_{2}=c_{2} \frac{\gamma+\gamma \circ \sigma}{2}+c_{2}^{\prime} \frac{\gamma-\gamma \circ \sigma}{2}
$$


D'ALEMBERT'S AND WILSON'S FUNCTIONAL EQUATIONS FOR VECTOR... 127

while (35) gives us that $G_{1}$ is a solution of the following "inhomogeneous" Wilson's functional equation

$$
\begin{aligned}
& \frac{G_{1}(x+y)+G_{1}(x+\sigma y)}{2}=G_{1}(x) \frac{\gamma+\gamma \circ \sigma}{2}(y) \\
& +G_{2}(x)\left\{\frac{\gamma+\gamma \circ \sigma}{2} a^{+}+\frac{\gamma-\gamma \circ \sigma}{2} a^{-}\right\}(y), x, y \in G .
\end{aligned}
$$

Direct computations show that

$$
c_{2} \frac{\gamma+\gamma \circ \sigma}{2} a^{+}+c_{2}^{\prime} \frac{\gamma-\gamma \circ \sigma}{2} a^{+}+c_{2} \frac{\gamma-\gamma \circ \sigma}{2} a^{-}+c_{2}^{\prime} \frac{\gamma+\gamma \circ \sigma}{2} a^{-}
$$

is a particular solution of (46), so that its complete solution is

$$
\begin{aligned}
G_{1} & =c_{2} \frac{\gamma+\gamma \circ \sigma}{2} a^{+}+c_{2}^{\prime} \frac{\gamma-\gamma \circ \sigma}{2} a^{+}+c_{2} \frac{\gamma-\gamma \circ \sigma}{2} a^{-}+c_{2}^{\prime} \frac{\gamma+\gamma \circ \sigma}{2} a^{-} \\
& +d_{1} \frac{\gamma+\gamma \circ \sigma}{2}+d_{2} \frac{\gamma-\gamma \circ \sigma}{2}
\end{aligned}
$$

where the last two terms as $d_{1}$ and $d_{2}$ range over $\mathrm{C}$ constitute the complete solution of the corresponding "homogeneous" Wilson's functional equation (see Theorem 2.2). We may use

$$
E_{4}=\gamma\left\{\begin{array}{cc}
1 & a^{+}+a^{-} \\
0 & 1
\end{array}\right\}, \alpha=\frac{1}{2}\left\{\begin{array}{l}
d_{1}+d_{2} \\
c_{2}+c_{2}^{\prime}
\end{array}\right\}, \beta=\frac{1}{2}\left\{\begin{array}{l}
d_{1}-d_{2} \\
c_{2}-c_{2}^{\prime}
\end{array}\right\} .
$$

Case (3): Here

$$
\Psi=\gamma^{+}\left\{\begin{array}{cc}
1 & a^{+}+S^{-} \\
0 & 1
\end{array}\right\}
$$

where $\gamma^{+} \in \mathscr{M}^{+}(G), a^{+} \in \mathscr{A}^{+}(G), S^{-} \in \mathscr{S}^{-}(G)$ and $a^{+}+S^{-} \neq 0$. We see from (37) that $G_{2}=\gamma^{+}\left(c_{2}+a_{2}^{-}\right)$, and from (35) that $G_{1}$ is a solution of

$$
\begin{aligned}
& \frac{G_{1}(x+y)+G_{1}(x+\sigma y)}{2} \\
& =G_{1}(x) \gamma^{+}(y)+G_{2}(x) \gamma^{+}(y)\left\{a^{+}(y)+S^{-}(y)\right\}, x, y \in G .
\end{aligned}
$$

Dividing the last identity by $\gamma^{+}(x+y)=\gamma^{+}(x) \gamma^{+}(y)=\gamma^{+}(x+\sigma y)$ we get for $G_{1}^{\prime}:=G_{1} / \gamma^{+}$that

$$
\begin{aligned}
& \frac{G_{1}^{\prime}(x+y)+G_{1}^{\prime}(x+\sigma y)}{2} \\
& =G_{1}^{\prime}(x)+\left(c_{2}+a_{2}^{-}(x)\right)\left\{a^{+}(y)+S^{-}(y)\right\}, \quad x, y \in G,
\end{aligned}
$$


i.e. $G_{1}^{\prime}$ satisfies the functional equation of symmetric second differences in product form. The solutions of this functional equation were given in Theorem 3.2. To find $G_{1}^{\prime}$ we go through the five cases (a)-(e) of Theorem 3.2.

Case (3)(a): Either $c_{2}+a_{2}^{-}=0$ or $a^{+}+S^{-}=0$. However, the last possibility is ruled out by the assumptions of this case (3). When $c_{2}+a_{2}^{-}=0$ then $G_{2}=0$. We find from Theorem 3.2 that $G_{1}=\gamma^{+}\left(c_{1}+a_{1}^{-}\right)$where $c_{1} \in \mathrm{C}$ and $a_{1}^{-} \in \mathscr{A}^{-}(G)$. We may use

$$
E_{5}=\gamma^{+}\left\{\begin{array}{cc}
1 & a^{+}+a_{1}^{-}+S^{-} \\
0 & 1
\end{array}\right\}, \alpha=\frac{1}{2}\left\{\begin{array}{c}
c_{1} \\
1
\end{array}\right\}, \beta=\frac{1}{2}\left\{\begin{array}{c}
c_{1} \\
-1
\end{array}\right\}
$$

(3)(b): $c_{2}+a_{2}^{-}$is a constant, i.e. $a_{2}^{-}=0$, so $G_{2}=c_{2} \gamma^{+}$. Theorem 3.2 gives

$$
G_{1}=\gamma^{+}\left[c_{2}\left(a^{+}+S^{-}\right)+a^{-}+c\right] .
$$

We may use

$$
E_{5}=\gamma^{+}\left\{\begin{array}{cc}
1 & a^{+}+a^{-}+S^{-} \\
0 & 1
\end{array}\right\}, \alpha=\frac{1}{2}\left\{\begin{array}{c}
c \\
c_{2}+1
\end{array}\right\}, \beta=\frac{1}{2}\left\{\begin{array}{c}
c \\
c_{2}-1
\end{array}\right\} .
$$

(3)(c): Here $\quad S^{-}=3 c\left(a_{2}^{-}\right)^{2}$ $G_{2}=\gamma^{+}\left(c_{2}+a_{2}^{-}\right)$ and $G_{1}=\gamma^{+}\left[c_{2} a^{+}+3 c_{2} c\left(a_{2}^{-}\right)^{2}+a^{+} a_{2}^{-}+c\left(a_{2}^{-}\right)^{3}+a_{1}^{-}+c_{1}\right]$. We may use

$$
\begin{aligned}
& E_{6}=\gamma^{+}\left\{\begin{array}{cc}
1+a_{2}^{-} & c\left(a_{2}^{-}\right)^{3}+3 c\left(a_{2}^{-}\right)^{2}+a^{+}+a^{+} a_{2}^{-}+a_{1}^{-} \\
0 & 1+a_{2}^{-}
\end{array}\right\}, \\
& \alpha=\frac{1}{2}\left\{\begin{array}{c}
c_{1} \\
c_{2}+1
\end{array}\right\}, \beta=\frac{1}{2}\left\{\begin{array}{c}
c_{1} \\
c_{2}-1
\end{array}\right\}
\end{aligned}
$$

Due to Lemma 3.5 below $c_{2}+a_{2}^{-}=0$ in the last two cases (d) and (e) of Theorem 3.2. That possibility was covered in Case (3)(a) above.

3.5. Lemma. Let $\gamma_{1}, \gamma_{2}, \cdots, \gamma_{N} \in \mathscr{M}(G)$ be $N$ different homomorphisms, and let $p_{1}, p_{2}, \cdots, p_{N}: G \rightarrow \mathrm{C}$ be algebraic combinations of additive functions. If $\sum_{i=1}^{N} p_{i} \gamma_{i}=0$ then $p_{1}=\cdots=p_{N}=0$.

Proof. This is Lemma 4.3 of [23].

Theorem 3.3 produces all solutions of the functional equation (30) for which $\operatorname{span}\left\{g(x) \in \mathrm{C}^{2} \mid x \in G\right\}=\mathrm{C}^{2}$ (see Proposition 3.1(b)). However, the dimension of $\operatorname{span}\left\{g(x) \in \mathrm{C}^{2} \mid x \in G\right\}$ need not be 2 . A non-trivial example comes about if, say, the second component of $g$ is identically 0 . So (30) has more solutions than the ones described in Theorem 3.3 if we allow degeneracy in $\Phi$. The following Corollary 3.6 describes all solutions of (30), also the new ones. 
3.6. Corollary. Each solution $(g, \Phi) \in C\left(G, \mathrm{C}^{2}\right) \times C\left(G, M_{2}(\mathrm{C})\right)$ of the functional equation (30) falls into one of the following 4 categories:

1. The solutions described in Theorem 3.3.

2. There exist $C \in \mathrm{GL}(2, \mathrm{C}), \gamma \in \mathscr{M}(G)$ with $\gamma \neq \gamma \circ \sigma$, and $\alpha_{1}, \beta_{1} \in \mathrm{C}$ such that

$$
\begin{aligned}
& C^{-1} g=\gamma\left(\begin{array}{c}
\alpha_{1} \\
0
\end{array}\right)+\gamma \circ \sigma\left(\begin{array}{c}
\beta_{1} \\
0
\end{array}\right), \\
& C=\frac{1}{2}(\gamma+\gamma \circ \sigma) I+\left(\begin{array}{cc}
0 & \psi_{1} \\
0 & \psi_{2}
\end{array}\right),
\end{aligned}
$$

where $\psi_{1}, \psi_{2} \in C(G)$ are arbitrary.

3. There exist $C \in \mathrm{GL}(2, \mathrm{C}), \gamma^{+} \in \mathscr{M}^{+}(G), a^{-} \in \mathscr{A}^{-}(G)$ and $c \in \mathrm{C}$ such that

$$
C^{-1} g=\left(c+a^{-}\right) \gamma^{+}\left(\begin{array}{l}
1 \\
0
\end{array}\right), \quad C^{-1} \Phi C=\gamma^{+} I+\left(\begin{array}{cc}
0 & \psi_{1} \\
0 & \psi_{2}
\end{array}\right),
$$

where $\psi_{1}, \psi_{2} \in C(G)$ are arbitrary.

4. $g=0$ and $\Phi$ is arbitrary in $C\left(G, M_{2}(\mathrm{C})\right)$.

Conversely, each of the pairs $g, \Phi$ described under (1)-(4) is a solution of the functional equation (30).

Proof. It is easy to check that all pairs $g, \Phi$ described under (1)-(4) are solutions of the functional equation (30), so it is left to show that each continuous solution $g, \Phi$ falls into one of the categories (1)-(4).

This has already been proved in Theorem 3.3 when $\operatorname{span}\{g(x) \in$ $\left.\mathrm{C}^{2} \mid x \in G\right\}=\mathrm{C}^{2}$ (see Proposition 3.1(2)), and it is a triviality that we get (4) when $\operatorname{span}\left\{g(x) \in \mathrm{C}^{2} \mid x \in G\right\}$ has dimension 0 so that $g=0$. Left is the case of $\operatorname{span}\left\{g(x) \in \mathrm{C}^{2} \mid x \in G\right\}=\mathrm{C} e$ for some $e \in \mathrm{C}^{2} \backslash\{0\}$. We write $g(x)=h(x) e, x \in G$, where $h \in C(G) \backslash\{0\}$. The functional equation (30) reveals that $\Phi(y)$ maps span $\left\{g(x) \in \mathrm{C}^{2} \mid x \in G\right\}$ into itself. In particular there exists $\phi \in C(G)$ such that $\phi(y) e=\Phi(y) e$ for all $y \in G$. When we substitute the expressions for $g$ and $\Phi$ above into (30) it becomes Wilson's scalar functional equation

$$
\frac{h(x+y)+h(x+\sigma y)}{2}=h(x) \phi(y), \quad x, y \in G,
$$

the solutions of which are written down in Theorem 2.2 above: There is $\gamma \in \mathscr{M}(G)$ such that $\phi=(\gamma+\gamma \circ \sigma) / 2$. If $\gamma \neq \gamma \circ \sigma$ then $h$ has the form $h=c(\gamma+\gamma \circ \sigma) / 2+d(\gamma-\gamma \circ \sigma) / 2$ where $c, d \in \mathrm{C}$. If $\gamma=\gamma \circ \sigma \in \mathscr{M}^{+}(G)$ then $h$ has the form $h=\left(c+a^{-}\right) \gamma$ where $c \in \mathrm{C}$ and $a^{-} \in \mathscr{A}^{-}(G)$. Letting $C$ denote an invertible $2 \times 2$ matrix such that $e=C\left(\begin{array}{l}1 \\ 0\end{array}\right)$ we find that 
$C^{-1} g(x)=C^{-1} h(x) e=h(x)\left(\begin{array}{l}1 \\ 0\end{array}\right)$, so $g$ has the form desired in (2) and (3). And from $\Phi(y) e=\phi(y) e$ we get that

$$
C^{-1} \Phi(y) C\left(\begin{array}{l}
1 \\
0
\end{array}\right)=\left(\begin{array}{c}
\phi(y) \\
0
\end{array}\right)
$$

implying the formula for $C^{-1} \Phi C$.

Let us for completeness of exposition write down the result analogous to Theorem 3.3 in which $\Phi(0)$ is a 1-dimensional projection instead of a 2-dimensional one.

3.7. Proposition. Let $\Phi: G \rightarrow M_{2}(\mathrm{C})$ be a continuous solution of the functional equation (6) and assume that $\Phi(0)$ is a 1-dimensional projection. Let $g=\left\{g_{1}, g_{2}\right\}^{t}: G \rightarrow \mathrm{C}^{2}$ be a continuous solution of (30). Then there are the following 2 possibilities:

1. There exist $C \in \mathrm{GL}(2, \mathrm{C}), \gamma \in \mathscr{M}(G)$ for which $\gamma \neq \gamma \circ \sigma$ and $c, c_{1}, c_{1}^{\prime} \in \mathrm{C}$ such that

$$
\begin{aligned}
C^{-1} g & =\left(c_{1}(\gamma+\gamma \circ \sigma) / 2+c_{1}^{\prime}(\gamma-\gamma \circ \sigma) / 2\right)\left\{\begin{array}{l}
1 \\
0
\end{array}\right\}, \\
C^{-1} \Phi C & =\left\{\begin{array}{cc}
(\gamma+\gamma \circ \sigma) / 2 & c(\gamma-\gamma \circ \sigma) / 2 \\
0 & 0
\end{array}\right\} .
\end{aligned}
$$

2. There exist $C \in \mathrm{GL}(2, \mathrm{C}), \gamma^{+} \in \mathscr{M}^{+}(G), c_{1} \in \mathrm{C}$ and $a^{-}, a_{1}^{-} \in \mathscr{A}^{-}(G)$ such that

$$
C^{-1} g=\left(\gamma^{+}\left(c_{1}+a_{1}^{-}\right)\right)\left\{\begin{array}{l}
1 \\
0
\end{array}\right\}, \text { and } C^{-1} \Phi C=\gamma^{+}\left\{\begin{array}{cc}
1 & a^{-} \\
0 & 0
\end{array}\right\} .
$$

Conversely, the formulas (61) and (62) define solutions of the functional equation (30).

Proof. We choose $C \in \mathrm{GL}(2, \mathrm{C})$ and $\gamma \in \mathscr{M}(G)$ as in Proposition 2.5. Consider $C^{-1} g=\left\{g_{1}, g_{2}\right\}^{t}$. If $\gamma \neq \gamma \circ \sigma$ we get from (22) that

$$
\begin{aligned}
& \frac{1}{2}\left\{\begin{array}{l}
g_{1}(x+y)+g_{1}(x+\sigma y) \\
g_{2}(x+y)+g_{2}(x+\sigma y)
\end{array}\right\}= \\
& \left\{\begin{array}{cc}
(\gamma+\gamma \circ \sigma) / 2 & c(\gamma-\gamma \circ \sigma) / 2 \\
0 & 0
\end{array}\right\}\left\{\begin{array}{l}
g_{1}(x) \\
g_{2}(x)
\end{array}\right\} .
\end{aligned}
$$

Proof. Putting $y=0$ in this identity we see that $g_{2}=0$, so that it reduces to

$$
\frac{g_{1}(x+y)+g_{1}(x+\sigma y)}{2}=g_{1}(x) \frac{\gamma+\gamma \circ \sigma}{2}(y), x, y \in G .
$$


i.e.the pair $\left\{g_{1}, \phi\right\}$ is a solution of the scalar Wilson's functional equation. We now refer to Theorem 2.2.

A similar argument works if $\gamma=\gamma \circ \sigma$.

\section{REFERENCES}

1. J. Aczél, Vorlesungen über Funktionalgleichungen und ihre Anwendungen, Birkhäuser 1961.

2. J. Aczél and J. Dhombres, Functional equations in several variables, Cambridge University Press, Cambridge - New York - New Rochelle - Melbourne - Sydney 1989.

3. R. Badora, On a joint generalization of Cauchy's and d'Alembert's functional equations, Aequationes Math. 43 (1992), 72-89.

4. J.A. Baker, The stability of the cosine equation, Proc. Amer. Math. Soc. 80 (1980), 411-416.

5. J.A. Baker and K.R. Davidson, Cosine, exponential and quadratic functions, Glasnik Matematički 16 (36) (1981), 269-274.

6. W. Chojnacki, Fonctions cosinus hilbertiennes bornées dans les groupes commutatifs localement compacts, Compositio Math. 57 (1986), 15-60.

7. W. Chojnacki, On some functional equation generalizing Cauchy's and d'Alembert's functional equations, Colloq. Math. 55 (1988), 169-178.

8. W. Chojnacki, Group representations of bounded cosine functions, J. Reine Angew. Math. 478 (1996), 61-84.

9. H.O. Fattorini, Uniformly bounded cosine functions in Hilbert space, Indiana Univ. Math. J. 20 (1970), 411-425.

10. Z. Gajda, A Generalization of D'Alembert's Functional Equation, Funkcial. Ekvac. 33 (1990), 69-77.

11. S. Kaczmarz, Sur l'équation fonctionelle $f(x)+f(x+y)=\phi(y) f\left(x+\frac{y}{2}\right)$, Fund. Math. 6 (1924), 122-129.

12. Pl. Kannappan, The functional equation $f(x y)+f\left(x y^{-1}\right)=2 f(x) f(y)$ for groups, Proc. Amer. Math. Soc. 19 (1968), 69-74.

13. J. Kisyński, On operator-valued solutions of d'Alembert's functional equation, I, Colloq. Math. 23 (1971), 107-114.

14. J. Kisyński, On operator-valued solutions of d'A

lembert's functional equation, II, Studia Math. 42 (1972), 43-66.

15. S. Kurepa, A cosine functional equation in Banach algebra, Acta Sci. Math. (Szeged) 23 (1962), 255-267.

16. S. Kurepa, Uniformly bounded cosine function in a Banach space, Math. Balkanica 2 (1972), $109-115$.

17. M.A. McKiernan, The matrix equation $a(x \circ y)=a(x)+a(x) a(y)+a(y)$, Aequationes Math. 15 (1977), 213-223.

18. P. Sinopoulos, Wilson's functional equation for vector and matrix functions, Proc. Amer. Math. Soc. 125 (1997), 1089-1094.

19. H. Stetkær, Wilson's Functional Equations on Groups, Aequationes Math. 49 (1995), 252275.

20. H. Stetkær, Functional Equations and Spherical Functions, Preprint Series 1994 No 18, Matematisk Institut, Aarhus University, Denmark. pp. 1-28.

21. H. Stetkær, Functional equations on abelian groups with involution, Aequationes Math. 54 (1997), 144-172.

22. L. Székelyhidi, Functional equations on abelian groups, Acta Math. Acad. Sci. Hungar. 37 (1981), 235-243. 
23. L. Székelyhidi, Convolution Type Functional Equations on Topological Abelian Groups, World Scientific. Singapore, New Jersey, London, Hong Kong. 1991.

24. W.H. Wilson, On certain related functional equations, Bull. Amer. Math. Soc. 26 (1919-20), $300-312$.

DEPARTMENT OF MATHEMATICS

AARHUS UNIVERSITY, BUILDING 530

DK 8000 AARHUS C

DENMARK

Email: stetkaer@imf.au.dk 